\title{
The geometrical theory of diffraction for axially symmetric reflectors
}

\author{
Rusch, W.; Sørensen, O.
}

Published in:

I E E E Transactions on Antennas and Propagation

Publication date:

1975

Document Version

Publisher's PDF, also known as Version of record

Link back to DTU Orbit

Citation (APA):

Rusch, W., \& Sørensen, O. (1975). The geometrical theory of diffraction for axially symmetric reflectors. IE E E Transactions on Antennas and Propagation, 23(3), 414-419.

\section{General rights}

Copyright and moral rights for the publications made accessible in the public portal are retained by the authors and/or other copyright owners and it is a condition of accessing publications that users recognise and abide by the legal requirements associated with these rights.

- Users may download and print one copy of any publication from the public portal for the purpose of private study or research.

- You may not further distribute the material or use it for any profit-making activity or commercial gain

- You may freely distribute the URL identifying the publication in the public portal

If you believe that this document breaches copyright please contact us providing details, and we will remove access to the work immediately and investigate your claim. 


$$
\begin{aligned}
& x_{3}=R \mp t-z-j b \mp j c \\
& x_{4}=R \mp t+z+j b \mp j c \\
& x_{5}=R \mp t \pm z \cos \psi .
\end{aligned}
$$

All quantities not defined here are defined in the main text.

\section{REFERENCES}

[1] H. E. King, "Mutual impedance of unequal length antennas in echelon," IRE Trans. Antennas Propagat., vol. AP-5, pp. 306-313, July 1957.

[2] J. H. Richmond and N. H. Geary, "Mutual impedance between coplanar-skew dipoles," IEEE Trans. Antennas Propagat. (Commun.), vol. AP-18, pp. 414-416, May 1970.

[3] J. H. Richmond, "Coupled linear antennas with skew orientation," IEEE Trans. Antennas Propagat. (Commun.), vol. AP-18, pp. 694-696, Sept. 1970

[4] - , "Admittance matrix of coupled V antennas," IEEE Trans. Antennas Propagat. (Commun.), vol. AP-18, pp. 820-821, Nov. 1970.

[5] - "Radiation and scattering by thin-wire structures in a homogeneous conducting medium," IEEE Trans. Antennas Propagat. (CPD), vol. AP-22, Mar. 1974, p. 365.

[6] - "Computer program for thin-wire structures in a homogeneous conducting medium," NASA Contractor Rep. CR-2399, June 1974, for sale by the National Technical Information Service, Springfield, Va. 22161 , price $\$ 3.75$.

[7] S. A. Schelkunoff and H. T. Friis, Antennas, Theory and Practice. New York: Wiley, 1952, pp. 370, 401 .

[8] D. V. Otto and J. H. Richmond, "Rigorous field expressions for piecewise-sinusoidal line sources," IEEE Trans. Antennas Propagat. (Commun.), vol. AP-17 p. 98, Jan. 1969.

\section{The Geometrical Theory of Diffraction for Axially Symmetric Reflectors}

W. V. T. RUSCH, SENIOR MEMBER, IEEE, AND O. SфRENSEN

Abstract-The geometrical theory of diffraction (GTD) (cf. [1], for example) may be applied advantageously to many axially symmetric reflector antenna geometries. The material in this communication presents analytical, computational, and experimental results for commonly encountered reflector geometries, both to illustrate the general principles and to present a compact summary of generally applicable formulas.

\section{AXIAlly Symmetric ReFLector for Vector SPHERICAL-Wave POINT SOURCE ON AXIS}

If a point-source feed is located on the axis of an axially symmetric reflector, then only two singly edge-diffracted rays are possible for a distant field point $P(R, \theta, \phi)$, if $\theta \neq 0, \pi$ (Fig. 1). These rays are "diffracted" from $Q_{E}{ }^{+}$, where the plane $\phi$ intersects the edge on the same side of the $Z$ axis as the field point, and from $Q_{E}{ }^{-}$, where the plane $\phi+\pi$ intersects the edge diametrically opposite $Q_{E}{ }^{+}$on the opposite side of the $Z$ axis from the field point.

The geometry of the edge-diffracted ray from $Q_{E}{ }^{-}$is shown in Fig. 2. (The surface is assumed to be convex.) The ray from the feed to $Q_{E}{ }^{-}$defines $\theta_{\text {edge, }}^{\prime}$ and the corresponding extreme geometrically reflected ray, when extended back to the $Z$ axis, defines $\theta_{\text {edge. }}$ These two angles may then be used to define two intermediate angles used in the edge-diffraction notation of

Manuscript received July 9, 1974; revised December 30, 1974

W. V. T. Rusch is with the Electromagnetics Institute, Technical University of Denmark, Lyngby, Denmark, on leave from the Department of Electrical Engineering, University of Southern California, Los Angeles, Calif. 90007 .

O. Sørensen is with the Electromagnetics Institute, Technical University of Denmark, Lyngby, Denmark.
J. B. Keller [2]:

$$
\begin{aligned}
& \delta_{\mathrm{t}}=\frac{\theta_{\mathrm{edge}}-\theta_{\mathrm{edge}}^{\prime}}{2} \\
& \alpha=-\frac{\theta_{\mathrm{edge}}+\theta_{\mathrm{edge}}^{\prime}}{2} .
\end{aligned}
$$

Let the total singly edge-diffracted field be defined by

$$
E_{d}=\left[E_{\mathrm{d} \theta}(\theta, \phi) a_{\theta}+E_{d \phi}(\theta, \phi) a_{\phi}\right] \frac{e^{-j k R}}{R}
$$

where the angles and unit vectors for both sets of feeds are defined in the sense indicated in Fig. 1. Then the components of $E_{d}$ can be decomposed into contributions from both $Q_{E}{ }^{+}$and $Q_{E}^{-}$

$$
\begin{aligned}
& E_{d \theta}(\theta, \phi)=E_{d \theta}{ }^{+}(\theta, \phi)+E_{d \theta}-(\theta, \phi) \\
& E_{d \phi}(\theta, \phi)=E_{d \phi}{ }^{+}(\theta, \phi)+E_{d \phi}{ }^{-}(\theta, \phi) .
\end{aligned}
$$

Considering first the diffracted field from $Q_{E}{ }^{-}$(because no transition functions are required for these rays)

$$
\begin{aligned}
& E_{d \theta}{ }^{-}(\theta, \phi) \\
& =\left\{-\frac{E_{f \theta}\left(\pi-\theta_{\text {edge }}^{\prime} \phi+\pi\right) e^{-j k \rho_{\text {edgo }}}}{\rho_{\text {edge }}}\right\} \\
& \cdot\left\{e^{J(\pi / 2)} \sqrt{\frac{D_{\text {refl }} / 2}{\sin \theta}}\right\} \\
& \text { - }\left\{\exp \left[j k\left[\left(2 c+Z_{\text {edge }}\right) \cos \theta-\left(D_{\text {refl }} / 2\right) \sin \theta\right]\right]\right\} D_{h}{ }^{-} \\
& E_{d \phi}{ }^{-}(\theta, \phi) \\
& =\left\{-\frac{E_{f \phi}\left(\pi-\theta_{\text {edge }}^{\prime} \phi+\pi\right) e^{-J k \rho_{\text {edse }}}}{\rho_{\text {edge }}}\right\} \\
& \left\{e^{j(\pi / 2)} \sqrt{\frac{D_{\mathrm{refl}} / 2}{\sin \theta}}\right\} \\
& \text { - }\left\{\exp \left[j k\left[\left(2 c+Z_{\text {edge }}\right) \cos \theta-\left(D_{\text {ref } 1} / 2\right) \sin \theta\right]\right]\right\} D_{s}{ }^{-}
\end{aligned}
$$

where $E_{f \theta}$ and $E_{f \phi}$ are the $\theta$ and $\phi$ components of the incident field, $\rho_{\text {edge }}$ is the distance from feed to $Q_{E}{ }^{-}, Z_{\text {edge }}$ is the $Z$ coordinate of $Q_{E}{ }^{-}\left(Z_{\text {cdge }}<0\right), D_{\text {refl }}$ is the reflector diameter, $Z=-2 c$ is an arbitrary phase reference point on the negative $Z$ axis, and the diffraction coefficients are given by

$$
\begin{aligned}
& \int\left\{-\frac{e^{-j(\pi / 4)}}{2 \sqrt{2 \pi k}}\left[\frac{1}{\cos \left(\theta+\delta_{t}+\alpha\right) / 2}\right.\right. \\
& \left.\left.\mp \frac{1}{\sin \left(\theta+\delta_{t}-\alpha\right) / 2}\right]\right\}, \quad 0<\theta \leq \frac{\pi}{2}-\delta_{t} \\
& D_{\substack{h \\
n^{-}}}=\left\{\begin{array}{l}
0, \quad \frac{\pi}{2}-\delta_{t}<\theta<\frac{\pi}{2} \\
\left\{-\frac{e^{-J(\pi / 4)}}{2 \sqrt{2 \pi k}}\left[-\frac{1}{\cos \left(\theta+\delta_{t}+\alpha\right) / 2}\right.\right. \\
\left.\left. \pm \frac{1}{\sin \left(\theta+\delta_{t}-\alpha\right) / 2}\right]\right\}, \quad \frac{\pi}{2} \leq \theta<\pi
\end{array}\right.
\end{aligned}
$$

where the upper signs are taken for the soft $(s)$ coefficient and the lower signs are taken for the hard (h) coefficient. Notice that 


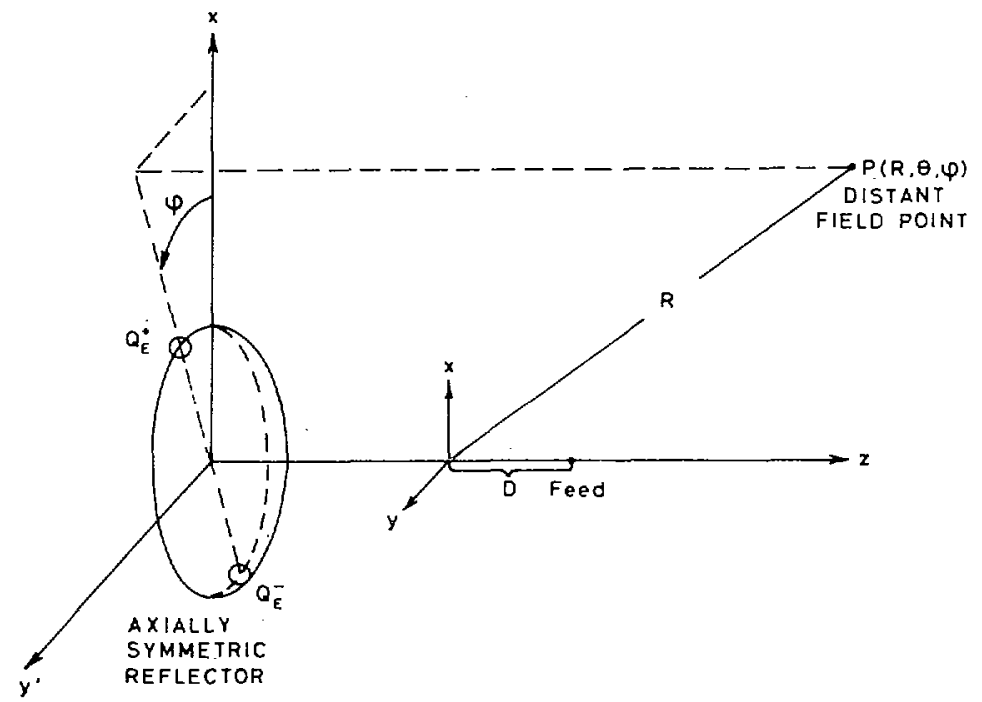

Fig. 1. Geometry of edge-diffraction points for axially symmetric reflector with feed on axis.

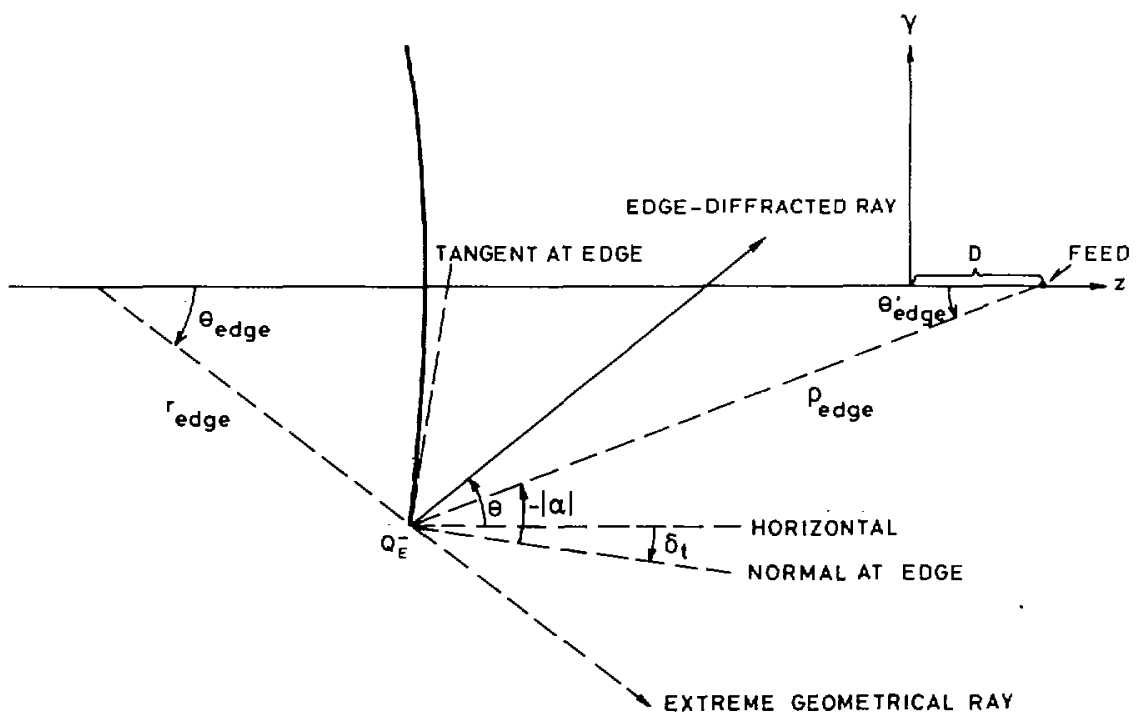

Fig. 2. Geometry of ray diffracted from $Q_{e}^{-}$.

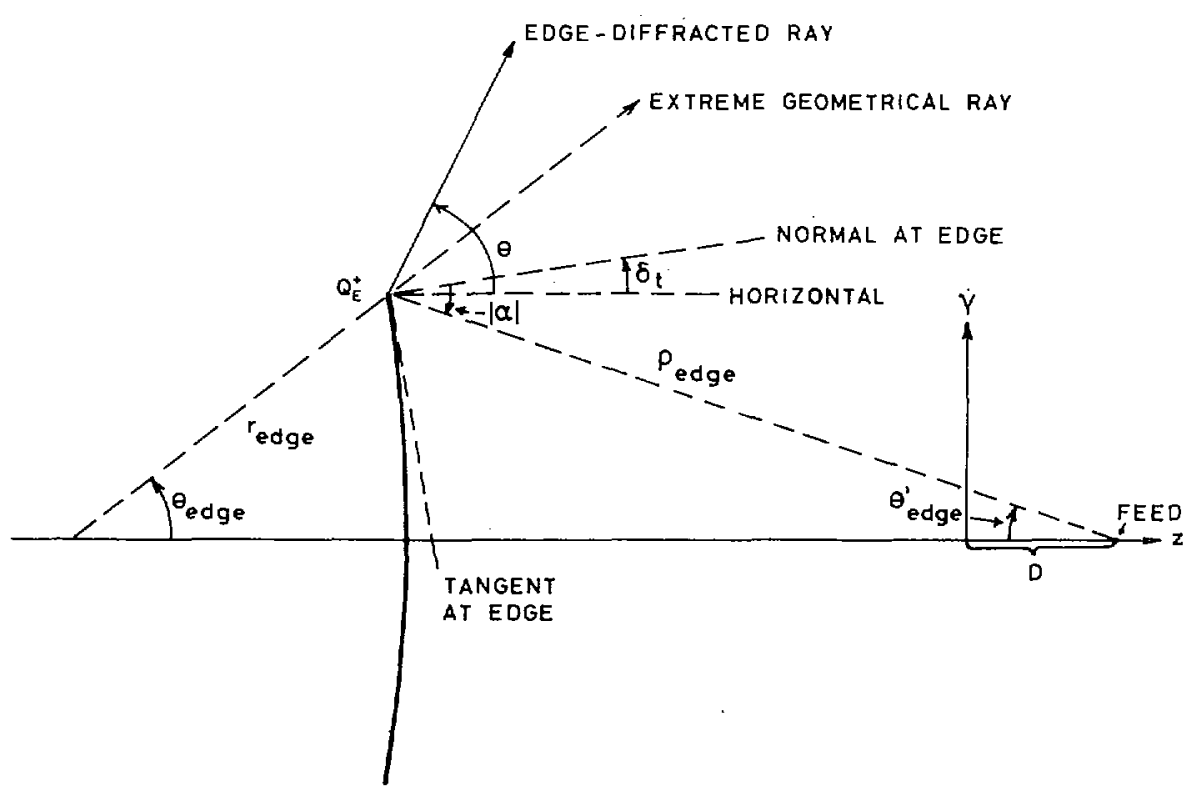

Fig. 3. Geometry of ray diffracted from $Q_{e}^{+}$. 
$D_{s}{ }^{-}$is zero in the direction of the surface tangent, $\theta=\pi / 2-\delta$, but $D_{h}{ }^{-}$is not. Slightly modified but principally the same diffraction coefficients for singly edge-diffracted rays may be derived for concave surfaces.

The geometry of the ray diffracted from $Q_{E}{ }^{+}$is shown in Fig. 3. The fields of this ray are given by

$$
\begin{aligned}
& E_{\mathrm{d} \theta}{ }^{+}\left(\theta_{,} \phi\right) \\
&=\left\{\frac{E_{f \theta}\left(\pi-\theta_{\text {edge, }}^{\prime} \phi\right) e^{-j k \rho_{\text {ed } g e}}}{\rho_{\text {edge }}}\right\}\left\{\sqrt{\frac{D_{\text {refl }} / 2}{\sin \theta}}\right\} \\
& \cdot\left\{\exp \left[j k\left[\left(2 c+Z_{\text {edge }}\right) \cos \theta+\left(D_{\text {ref } 1} / 2\right) \sin \theta\right]\right]\right\} D_{h}^{+}
\end{aligned}
$$

$$
\begin{aligned}
E_{d \phi}+(\theta, \phi) & \\
= & \left\{\frac{E_{f \phi}\left(\pi-\theta_{\text {edge }}^{\prime}, \phi\right) e^{-j k \rho_{\text {edge }}}}{\rho_{\text {edge }}}\right\}\left\{\sqrt{\frac{D_{\text {ref } 1 / 2}}{\sin \theta}}\right\} \\
& \cdot\left\{\exp \left[j k\left[\left(2 c+Z_{\text {edge }}\right) \cos \theta+\left(D_{\text {ref } 1} / 2\right) \sin \theta\right]\right]\right\} D_{\mathrm{s}}^{+}
\end{aligned}
$$

where [1]

$$
\begin{aligned}
& D_{s}^{+}=\left\{-\frac{e^{-j(\pi / 4)}}{2 \sqrt{2 \pi k}}\left[\frac{F\left[k L^{l} a\left(\theta-\delta_{t}-\alpha\right)\right]}{\cos \left(\theta-\delta_{t}-\alpha\right) / 2}\right.\right. \\
& \left.\left. \pm \frac{F\left[k L^{r} a\left(\pi+\theta-\delta_{t}+\alpha\right)\right]}{\sin \left(\theta-\delta_{t}+\alpha\right) / 2}\right]\right\} \\
& a(x) \equiv 2 \cos ^{2}\left(\frac{x}{2}\right) \\
& F(k L a)=2 j \sqrt{k L a} e^{j k L a} \int_{\sqrt{k L a}}^{\infty} e^{-j \tau^{2}} d \tau .
\end{aligned}
$$

The transition functions $F(k L a)$ remove the singularities at the shadow boundary $\left(\theta=\pi-\theta_{\text {edge }}^{\prime}\right)$ and at the reflection boundary $\left(\theta=\theta_{\text {edge }}\right)$. Furthermore,

$$
\begin{aligned}
& L^{L}=\rho_{\text {edge }} \\
& L^{r}=\frac{\beta_{1}{ }^{r} \beta_{2}{ }^{r} \sin \theta_{\text {edge }}}{\left(D_{\text {refl }} / 2\right)}
\end{aligned}
$$

where $\beta_{1}{ }^{r}$ and $\beta_{2}{ }^{r}$ are the principal radii of curvature of the reflected geometrical ray at $Q_{E}{ }^{+}$and $\left(D_{\text {ref }} / 2\right) / \sin \theta_{\text {cdge }}$ is the caustic distance evaluated in the direction of the reflection boundary.

For the point source on the $Z$ axis illuminating the axially symmetric reflector, the resulting single edge-diffracted rays have a caustic at all points on the $Z$ axis. Thus all ray path-lengths $\overline{F Q_{E} P}$ are the same if both $F$ and $P$ lie on the axis of symmetry. Ray optical solutions fail in the neighborhood of these caustics, as, for example, the $\sin \theta$ denominator in the square-root factor of (4) and (6) vanishes. Alternative solutions near caustics are canonical solutions [2], asymptotic solutions [3], [4], and integral solutions [1], [5]. The latter technique consists of replacing the edge-diffracted rays with contributions from equivalent electric and magnetic ring currents lying along the edge:

$$
\begin{aligned}
& I_{e \phi} \cong-\frac{E_{\phi} D_{s}\left(\phi, \phi^{\prime} ; \pi / 2\right)}{\eta} \sqrt{\frac{8 \pi}{k}} e^{-j \pi / 4} \\
& I_{m \phi}=-H_{\phi} \eta D_{h}\left(\phi, \phi^{\prime} ; \pi / 2\right) \sqrt{\frac{8 \pi}{k}} e^{-j \pi / 4}
\end{aligned}
$$

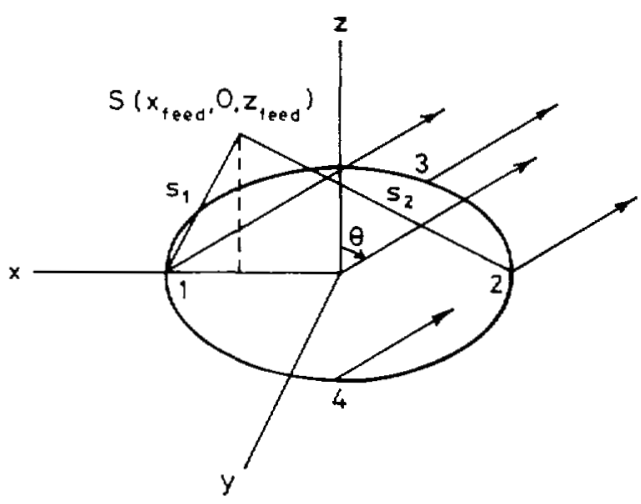

Fig. 4. Geometry of four possible edge rays.

where $E_{\phi}$ and $H_{\phi}$ are the edge-tangential incident electric and magnetic fields, $D_{s}\left(\phi, \phi^{\prime} ; \pi / 2\right)$ and $D_{h}\left(\phi, \phi^{\prime} ; \pi / 2\right)$ are the soft and hard edge-diffraction coefficients, and $\phi, \phi^{\prime}$ are the angles of diffraction and incidence in the "Ray-fixed" coordinate systems defined by Kouyoumjian [1]. For an arbitrary field point $\phi$ is different at various points on the rim. However, for a field point on axis $\phi$ is constant (at great distances on axis $\phi=\pi / 2-\delta_{t}$. Furthermore, for this symmetric case $\phi^{\prime}=\pi / 2+\alpha$ ).

The most commonly encountered feed function is that of a far-field, $m=1$, spherical-wave source for which the tangential fields incident on the edge are

$$
\begin{aligned}
& E_{\phi}=d_{1}\left(\theta_{s}\right) \cos \phi_{s} \frac{e^{-j k \rho_{\text {edge }}}}{\rho_{\text {edge }}} \\
& H_{\phi}=\frac{1}{\eta} a_{1}\left(\theta_{s}\right) \sin \phi_{s} \frac{e^{-j k \rho_{\text {edge }}}}{\rho_{\text {edge }}} .
\end{aligned}
$$

The easily computed ring current fields then become as follows. $\theta=0$ :

$$
\begin{aligned}
E_{\text {ring }}= & a_{y} \frac{\left(D_{\text {refl } 1} / 2\right)}{4} \exp \left[j k\left(2 c+Z_{\text {edge }}\right)\right] \frac{e^{-j k R}}{R} \\
& \cdot\left\{-\left[d_{1}\left(\pi-\theta_{\text {edge }}^{\prime}\right) \frac{e^{-j k \rho_{\text {edge }}}}{\rho_{\text {edge }}}\right]\right. \\
& \cdot\left[\frac{1}{\cos \left(\theta_{\text {edge }}^{\prime} / 2\right)}-\frac{1}{\sin \left(\theta_{\text {edge }} / 2\right)}\right] \\
& -\left[a_{1}\left(\pi-\theta_{\text {edge }}^{\prime}\right) \frac{e^{-j k \rho_{\text {edge }}}}{\rho_{\text {edge }}}\right] \\
& \left.\cdot\left[\frac{1}{\cos \left(\theta_{\text {edge }}^{\prime} / 2\right)}+\frac{1}{\sin \left(\theta_{\text {edge }} / 2\right)}\right]\right\}
\end{aligned}
$$

$\theta=\pi$

$$
\begin{aligned}
\boldsymbol{E}_{\mathrm{ring}}= & a_{y} \frac{\left(D_{\mathrm{ref} 1} / 2\right)}{4} \exp \left[-j k\left(2 c+Z_{\mathrm{edge}}\right)\right] \frac{e^{-j k \boldsymbol{R}}}{R} \\
\cdot & \left\{-\left[d_{1}\left(\pi-\theta_{\mathrm{edge}}^{\prime}\right) \frac{e^{-j k \rho_{\text {edge }}}}{\rho_{\text {edge }}}\right]\right. \\
\cdot & {\left[\frac{1}{\cos \left(\theta_{\mathrm{edge}} / 2\right)}-\frac{1}{\sin \left(\theta_{\mathrm{edge}}^{\prime} / 2\right)}\right] } \\
& -\left[a_{1}\left(\pi-\theta_{\mathrm{edge}}^{\prime}\right) \frac{e^{-j k \rho_{\text {edge }}}}{\rho_{\text {edge }}}\right] \\
\cdot & {\left.\left[\frac{1}{\cos \left(\theta_{\mathrm{edge}} / 2\right)}+\frac{1}{\sin \left(\theta_{\text {edge }}^{\prime} / 2\right)}\right]\right\} . }
\end{aligned}
$$


$\theta$ small but nonzero:

$$
\begin{aligned}
E_{\text {rigg }} \cong & \frac{\left(D_{\text {ref1 }} / 2\right)}{4} \exp \left[j k\left(2 c+Z_{\text {edge }}\right) \cos \theta\right] \frac{e^{-j k \rho_{\text {edge }}}}{\rho_{\text {edge }}} \frac{e^{-j k R}}{R} \\
& \cdot\left\{a _ { \theta } \left[-d_{1}\left(\pi-\theta_{\text {edge }}^{\prime}\right)\left[\frac{1}{\cos \left(\theta_{\text {edge }}^{\prime} / 2\right)}\right.\right.\right. \\
& \left.-\frac{1}{\sin \left(\theta_{\text {edge }} / 2\right)}\right]\left[J_{0}(\beta)+J_{2}(\beta)\right] \cos \theta \\
& -a_{1}\left(\pi-\theta_{\text {edge }}^{\prime}\right)\left[\frac{1}{\cos \left(\theta_{\text {edge }}^{\prime} / 2\right)}+\frac{1}{\sin \left(\theta_{\text {edge }} / 2\right)}\right] \\
& \left.\cdot\left[J_{0}(\beta)-J_{2}(\beta)\right]\right] \sin \phi \\
& +a_{\phi}\left[-d_{1}\left(\pi-\theta_{\text {edge }}^{\prime}\right)\left[\frac{1}{\cos \left(\theta_{\text {edge }}^{\prime} / 2\right)}\right.\right. \\
& \left.-\frac{1}{\sin \left(\theta_{\text {edge }} / 2\right)}\right]\left[J_{0}(\beta)-J_{2}(\beta)\right] \\
& -a_{1}\left(\pi-\theta_{\text {edge }}^{\prime}\right)\left[\frac{1}{\cos \left(\theta_{\text {edge }}^{\prime} / 2\right)}+\frac{1}{\sin \left(\theta_{\text {edge }} / 2\right)}\right] \\
& \left.\left.\cdot\left[J_{0}(\beta)+J_{2}(\beta)\right] \cos \theta\right] \cos \phi\right\}
\end{aligned}
$$

where $\beta=k\left(D_{\text {ref1 }} / 2\right) \sin \theta$. Equation (12c) is an approximation in that the changes in $D_{s}$ and $D_{h}$ in equations (10a) and (10b) at various points on the rim have been assumed to be second order for relatively small values of $\theta$.

\section{AXIALLY SYMMETRIC REFLECTORS FOR \\ Vector Spherical-Wave PoINT Source OFf Axis}

If a point-source feed is located off the axis of the axially symmetric reflector, Fermat's principle reveals that there are a maximum of four possible diffraction points around the rim of the reflector [6]. The situation becomes somewhat less complicated when the point-source feed, the reflector axis, and the observation point all lie in the same plane (taken, for example, to be the $X-Z$ plane in Fig. 4). Under these conditions, diffraction points 1 and 2 are possible for all possible positions of the distant field point. However, if the field point is on the opposite side of the axis from the source point, then two additional diffraction points 3 and 4 are also possible in the range of $\theta$ values, $\theta_{1} \leq \theta \leq \theta_{2}$, [7] where

$$
\begin{aligned}
& \theta_{1}=\sin ^{-1}\left[\frac{X_{\text {feed }}}{\sqrt{\left(D_{\text {ref1 } 1} / 2+X_{\text {feed }}\right)^{2}+\left(Z_{\text {edge }}-Z_{\text {feed }}\right)^{2}}}\right] \\
& \theta_{2}=\sin ^{-1}\left[\frac{X_{\text {feed }}}{\sqrt{\left(D_{\text {refl } 1} / 2+X_{\text {feed }}\right)^{2}+\left(Z_{\text {edge }}-Z_{\text {feed }}\right)^{2}}}\right]
\end{aligned}
$$

$\theta_{1}$ corresponds to a caustic at infinity form point 2 , while $\theta_{2}$ corresponds to a caustic at infinity from point 1 . Thus, as $\theta$ increases from $\theta_{1}$ to $\theta_{2}$, the additional roots 3 and 4 move from 2 to 1 . In practice, for typical reflector geometries the range of values from $\theta_{1}$ to $\theta_{2}$ is sufficiently small so that the entire range is covered by radiation from the equivalent ring sources to avoid unrealistically large predicted fields. An example of a multicaustic region is for values of $\theta$ between $\theta_{1}$ and $\theta_{2}$ in the plane of scan as considered previously. Under these conditions it is necessary to account for the variations of $E_{\phi}, H_{\phi}, D_{s}$, and $D_{h}$ by evaluating the radiation integrals numerically. Examples of typical results are shown in Fig. 5 for a laterally defocused

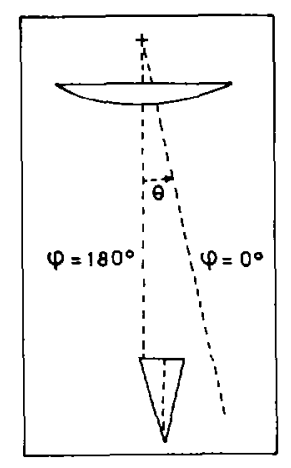

$x--x-4 x$ Ghysical Optics $x--x--x$ Geometrical Optics + GTD 0 - -0 Geometrical Optics. Ring Currents
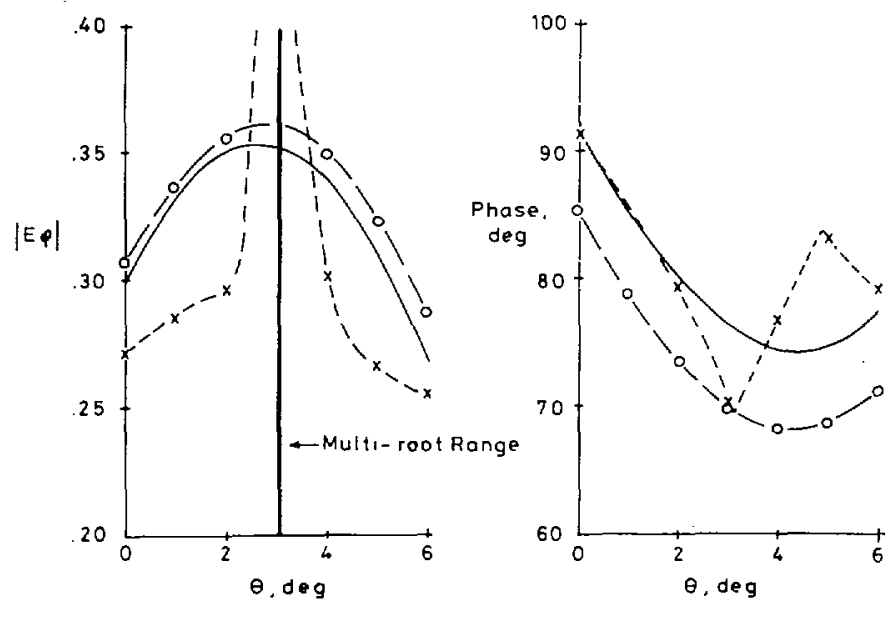

$\Delta x=3 \mathrm{~cm} \quad \varphi=180^{\circ}$

Fig. 5. Comparison of PO, GTD, and ring-current fields in multicaustic directions.

hyperboloid. On the opposite side of the axis in the plane of scan the multiroot range lies between 3.01 and $3.11 \mathrm{deg}$. However, the simple geometrical theory of diffraction (GTD) two-edge ray description of the field is seen to become unrealistically large outside the multiroot range. The ring-current contribution, on the other hand, is well behaved and very close to the physical optics results over a relatively wide angular range.

\section{SURface Diffracted Rays}

First-order surface rays will ordinarily not be directly excited by the feed in a reflecting antenna system. However, they may be excited by tangential edge-diffracted rays. An example may be seen in Fig. 2 where an edge-diffracted ray from $Q_{E}{ }^{-}$in the direction $\theta=\pi / 2-\delta_{t}$ will tangentially graze the surface. Only the tangential $H$-field component will be nonzero in this direction, but the excited surface rays are necessary to provide a continuous field. In most instances, however, the edge-diffracted rays provide sufficiently accurate surface rays.

\section{APPLICATIONS}

\section{A. Radiation from Prime-Focus Paraboloid}

The Kouyoumjian group has used a combination of a single edge-diffracted GTD, equivalent ring-currents, and physical optics to compute the complete radiation pattern of a prime focus paraboloid [8], [9]. The calculated pattern was compared with the experimental results of Afifi, which were measured for a halfparaboloid on a groundplane with a monopole feed at the focus [10]. This experimental arrangement virtually eliminated aperture 


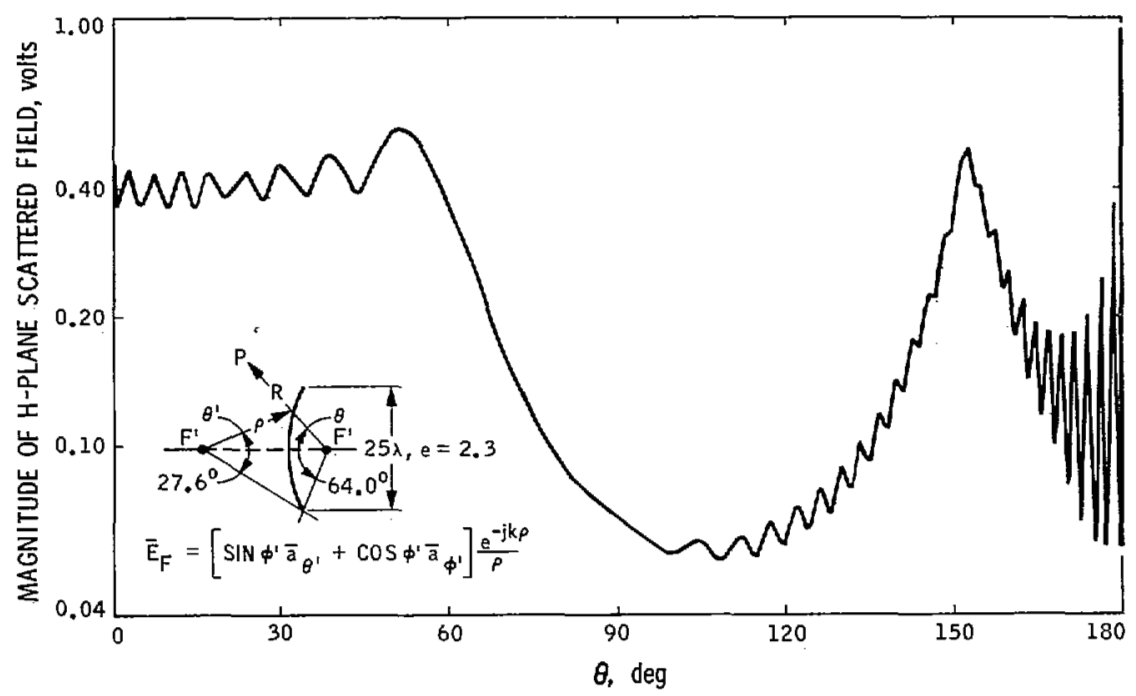

Fig. 6. Complete GTD $H$-plane pattern of hyperboloid with feed at external focus.

blocking. The vertical polarization eliminated the possibility of exciting surface-diffracted rays. The deep reflector $(F / D=0.25)$ eliminated rear spillover. The agreement between the calculated and measured patterns was very good between $0 \mathrm{deg}$ and $130 \mathrm{deg}$. Beyond $130 \mathrm{deg}$ the agreement was also quite good, considering that at such low signal levels part of the discrepancy was due to measurement error.

\section{B. Radiation from Hyperboloid with Spherical-Wave Feed at Its External Focus}

Results have been obtained for a "focused" hyperboloid using physical optics, geometrical optics, GTD (Keller), and GTD (Kouyoumjian) [11]. The geometrical-optics results are discontinuous at the reflection boundary. The physical optics and Kouyoumjian GTD results agree closely in magnitude and phase, both in the illuminated and shadowed regions as well as in the vicinity of the reflection boundary.

Fig. 6 is a complete $H$-plane pattern based on GTD. The fields at the reflection boundary at $64 \mathrm{deg}$ and the shadow boundary at $152.4 \mathrm{deg}$ are finite and continuous, and the two axial caustics are computed using the equivalent ring sources. This technique yields slightly different results at the rear axial caustic than the caustic correction factor of Keller [2], which did not yield $E$-plane- $H$-plane continuous fields on axis.

Results were also reported in [11] that compare the calculated and measured scattered $H$-plane patterns from a hyperboloid illuminated by a corrugated horn with its phase center at the hyperboloid's external focus [12]. The GTD results included two edge rays from the reflector and one from the leading edge of the horn on the field-point side of the axis. Equivalent ring currents around both the reflector and horn rims were used for field points near the axis. These GTD results agreed with the measured patterns within the experimental uncertainties.

\section{Evaluation of Focused Equivalent Parabolold USING GTD}

The techniques of GTD provide a computational procedure to obtain RF performance data that hitherto was excessively costly in computer time. A prominent example of this is the dual-reflector antenna. Hitherto, it was only possible to analyze dual-reflector systems by integrating over both reflectors. The subreflector in a system of this type generally creates a shaped,

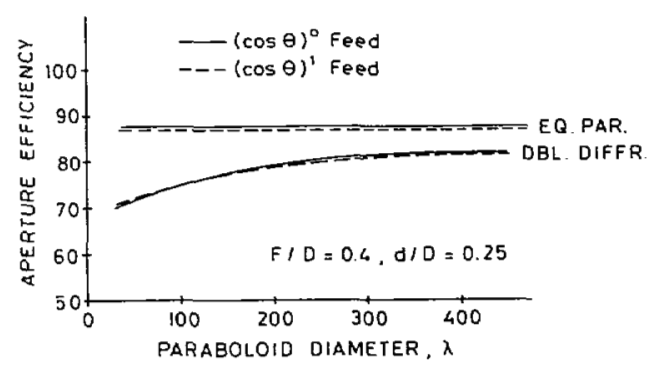

Fig. 7. Comparison of aperture efficiency values using GTD and equivalent paraboloid.

rather than a focused, beam and consequently is readily amenable to GTD determination of the scattered field. This rapidly determined scattered field then provides the illumination function for a physical optics integration over the large focused primary mirror.

The equivalent paraboloid is said to provide an accurate technique for the calculation of performance characteristics for Cassegrain and Gregorian systems [13]. GTD provides an accurate and relatively economical technique to verify this assertation quantitatively. For example, in Fig. 7, the aperture efficiency (exclusive of spillover) is calculated for a Cassegrain system with a paraboloid $F / D=0.4$ and subreflector diameter/main reflector diameter ratio $=0.25$. Diffraction from the subreflector causes the illumination of the main reflector to be tapered. However, this taper is not included in the equivalent paraboloid calculation, and its predicted efficiency is significantly higher, even for subdish diameters as large as 100 wavelengths. Since the edge illumination of the equivalent paraboloid is higher than the actual edge illumination, it generally predicts higher wide sidelobes than actually exist [14].

\section{REFERENCES}

[1] R. G. Kouyoumjian and P. H. Pathak, "A uniform geometrical theory of diffraction for an edge in a perfectly conducting surface," Proc. $I E E E$, vol. 62, po. 1448-1461, Nov. 1974.

[2] J. B. Keller, "Diffraction by an aperture,". J. Appl. Phys., vol 28,

[3] 1. Kay and J. B. Keller, "Asymptotic evaluation of the field at a caustic," J. Appl. Phys., vol. 25, pp. 876-883, July 1954.

[4] D. Ludwig, "Uniform asymptotic expansions at a caustic," Commun. Pure Appl. Math., vol. 19, pp. 215-250, May 1966. 
[5] G. L. James and V. Kerdemelidis, "Reflector antenna radiation pattern analysis by equivalent edge currents," IEEE Trans. Antennas Propagat., vol. AP-21, pp. 19-24, Jan. 1973.

[6 F. Molinet and L. Saltiel, "High frequency radiation pattern prediction for satellite antennas," Lab. Central de Télécommunications, ESTEC Contract 1820/72HP, July 1973 .

[7] H. Bach, K. Pontoppidan and, L. Solymar, "High frequency radiation pattern prediction for satellite antennas," Lab. Electromagnetic Theory, Tech. Univ. Denmark, Lyngby, ESTEC Contract 1821/72HP, Dec. 1973.

[8] R. G. Kouyoumjian and P. A. J. Ratnasiri, "The calculation of the complete pattern of a reflector antenna," in Proc. Int. Electronics Conf., 1969, pp. 152-153.

[9] P. A. J. Ratnasiri, R. G. Kouyoumjian, and P. H. Pathak, "The wide angle sidelobes of reflector antennas," Electro Sci. Lab. Ohio State Univ., Columbus, Rep. 2183-1, 1970.

[10] M. S. Afifi, "Radiation from the paraboloid of revolution," in Electromagnetic Wave Theory, part 2, J. Brown, Ed. New York: Pergamon, 1967, pp. 669-687.

[11] W. V. T. Rusch, "A comparison of geometrical and integral fields from high-frequency reflectors," Proc. IEEE (Lett.), vol. 62, pp. 16031604, Nov. 1974.

[12] O. Sorensen, "Analysis of defocused Cassegrain antennas using geometrical theory of diffraction," Master's thesis, Lab. Electromagnetic Theory, Tech. Univ. Denmark, Lyngby, Fall 1973.

[13] W. C. Wong, "On the equivalent parabola technique to predict the performance characteristics of a Cassegrainian system with an offset feed," IEEE Trans. Antennas Propagat., vol. AP-21, pp. 335-339, Mayt 1973.

[14] R. Booth, private communication.

\section{Relation Between the Radiation Pattern of an Array and the Two-Dimensional Discrete Fourier Transform}

\section{S. HAYKIN AND J. KESLER}

Abstract-A linear wideband array with each element followed by a tapped-delay line may be considered as a two-dimensional digital filter. Accordingly, the radiation pattern of the processor may be expressed as the product of a pair of two-dimensional discrete Fourier transforms (DFT's).

\section{INTRODUCTION}

It is well known that $z$ transform relations in linear systems can be used to describe the behavior of linear antenna arrays [1]. The objective of this article is to extend this treatment to a wideband array processor consisting of a linear array, each

$$
W\left(z_{1}, z_{2}\right)=\left[\begin{array}{lll}
w_{00} & +w_{01} z_{2}^{-1} & +\cdots+w_{0, M-1} z_{2}^{-(M-1)} \\
+w_{10} z_{1}-1 & +w_{11} z_{1}^{-1} z_{2}-1 & +\cdots+w_{1, M-1} z_{1}^{-1} z_{2}^{-(M-1)} \\
+w_{N-1,0} z_{1}^{-(N-1)} & +w_{N-1,1} z_{1}^{-(N-1)} z_{2}^{-1} & +\cdots+w_{N-1, M-1} z_{1}^{-(N-1)} z_{2}^{-(M-1)}
\end{array}\right] \text {. }
$$

element of which is followed by a tapped-delay line network [2]. It will be shown that the radiation pattern of such a processor can be expressed as the product of a pair of two-dimensional discrete Fourier transforms (DFT's).

\section{The Interpretation of an ARray Processor as a Two-Dimensional Digrtal Foter}

A linear antenna array may be viewed as a sampled data system in the sense that the current distribution in the discrete elements of the array may be considered as the sampled values of a continuous function [1]. The equivalent sampling period of

Manuscript received November 17, 1974.

The authors are with the Communications Research Laboratory, Faculty of Engineering, McMaster University, Hamilton, Ont., Canada.

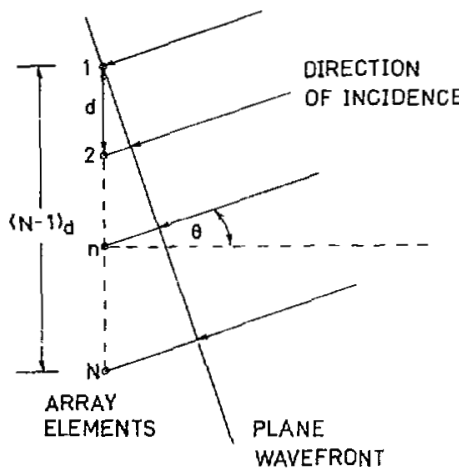

Fig. 1. Linear array.

the array is

$$
t_{d}=\frac{d \sin \theta}{\lambda f}
$$

where, according to Fig. 1, $d$ is the spacing between elements, $\theta$ is the direction of incidence of the plane wavefront impinging on the array elements, and $\lambda$ and $f$ are the wavelength and frequency, respectively. The $N$ elements of the array are assumed to be isotropic point sources radiating uniformly in all directions with equal amplitude and phase.

Consider a wideband array processor in which the received signals are delayed, weighted, and summed as shown in Fig. 2. The tapped-delay line connected to each element permits adjustment of gain and phase as desired at a number of frequencies over the frequency band of interest. Thus, this system can perform filtering in both the space and time domains. In general, time delay $\tau$ is different from the equivalent sampling period $t_{d}$.

Assuming that the array processor consists of $N$ radiating elements and $(M-1)$ delay elements, as in Fig. 2, the values of the weights in the processor form a two-dimensional $N \times M$ matrix, as shown by

$$
W=\left[\begin{array}{cccc}
w_{00} & w_{01} & \cdots & w_{0, M-1} \\
w_{10} & w_{11} & \cdots & w_{1, M-1} \\
\vdots & & & \\
w_{N-1,0} & w_{N-1,1} & \cdots & w_{N-1, M-1}
\end{array}\right]
$$

Define a polynomial $W\left(z_{1}, z_{2}\right)$ in the two variables $z_{1}=$ $\exp \left(j \omega t_{d}\right)$ and $z_{2}=\exp (j \omega \tau)$, as follows:

The polynomial $W\left(z_{1}, z_{2}\right)$ may be expressed in the form of a twodimensional $z$ transform,

$$
W\left(z_{1}, z_{2}\right)=\sum_{n=0}^{N-1} \sum_{m=0}^{M-1} w_{n m} z_{1}^{-n} z_{2}{ }^{-m}
$$

Given an $N \times M$ input data matrix of sample values defined by

$$
\boldsymbol{X}=\left[\begin{array}{cccc}
x_{00} & x_{01} & \cdots & x_{0, M-1} \\
x_{10} & x_{11} & \cdots & x_{1, M-1} \\
\vdots & & \cdots & \\
x_{N-1,0} & x_{N-1,1} & \cdots & x_{N-1, M-1}
\end{array}\right]
$$

its $z$ transform is

$$
X\left(z_{1}, z_{2}\right)=\sum_{n=0}^{N-1} \sum_{m=0}^{M-1} x_{n m_{1}}{ }^{-n} z_{2}{ }^{-m} .
$$

